\title{
Heat Flow Field Analysis of the Effects of Vertical Piezoelectric Fan Placement on Heat Convection in Pin-Fin Heat Sink
}

\author{
Ya-Chu Chang, ${ }^{*}$ Go-Long Tsai, ${ }^{1}$ and Yen-Yeh Chueh ${ }^{1}$ \\ Graduate Institute of Mechanical and Electrical Engineering, National Taipei University of Technology \\ 1, Sec. 3, Zhongxiao E. Rd., Taipei 10608, Taiwan, R.O.C. \\ 'Department of Vehicle Engineering, National Taipei University of Technology \\ 1, Sec. 3, Zhongxiao E. Rd., Taipei 10608, Taiwan, R.O.C.
}

(Received August 30, 2016; accepted December 21, 2016)

Keywords: electronic cooling, computational fluid dynamics, horizontal piezoelectric fan, heat sink

Piezoelectric fans make use of the inverse piezoelectric effect to actuate the rotation of blades. These fans have the advantages of small size, low power consumption, and low noise output. In this study, we used the commercial computational fluid dynamics (CFD) software ANSYS CFD/ Fluent to simulate a transient flow field in order to explore the impact of each column on the heat flow of a rectangular-channel piezoelectric fan installed inside each columnar radiator. The aim of this study was to find the best position for the piezoelectric fan cooling radiator. In the numerical simulation, the pressure radiator fan blade tip-to-tip distance $\left(L_{g}\right)$, height of the piezoelectric fan center from the flow channel plate $\left(H_{w}\right)$, fin row $(n)$, radiator fin width $\left(W_{f}\right)$, and the fin gap $(G)$ were used for the vertical piezoelectric fan to obtain the Niu Saier number $\left(N_{u}\right)$ and thermal resistance $\left(R_{t h}\right)$. Cyclical swings of the piezoelectric fan impede the uninterrupted flow of impact energy transfer on the boundary layer. At the tip of the blade actuation direction, the fluid reciprocating stroke, that is, the direction of the flow field, was pulled, and the front tip of the blade generated a vortex forming an entrained flow, but the hot and cold air were effectively mixed. The results showed that when the height of the center of the piezoelectric fan in relation to the flow channel plate was increased, the dissipation performance decreased comparatively. Furthermore, when a pressure fan was placed in front of the radiator, the height from the base center to the flow channel of the piezoelectric fan was reduced.

\section{Introduction}

With the considerable progress and development in semiconductor fabrication processes, electronic components are becoming smaller and more lightweight, making the issues of overheating increasingly challenging. Within the same wafer size, the number of transistors in the internal integrated circuit (IC) of a CPU increases with each advancement in fabrication processes, which means that the heat produced by each wafer also increases. To maintain rapid chip advancement and prevent chip failure at high temperatures, cooling mechanisms and technology for electronic devices are vital to the industry.

"Corresponding author: e-mail: changyachu0937@gmail.com http://dx.doi.org/10.18494/SAM.2017.1512 
Owing to their convenience and low cost, heat sinks are only added to a number of thermal management systems in order to reduce the heat of a chip. ${ }^{(1-5)}$ Unfortunately, the cooling effects of heat sinks without fans are limited. Forced convection is another choice. In addition to traditional rotary blade fans, piezoelectric fans are another option for better forced heat convection and surface heat transfer via elastic cantilever and blade vibration.

Acikalin et al.(6) discovered that the natural convection heat transfer coefficient increased by $100 \%$ using piezoelectric fans. They conducted experiments using a mobile phone with a $40 \times 5.7$ $\times 3.8 \mathrm{~cm}^{3}$ flow channel. In this experiment, the fan size was $6.35 \times 1 \mathrm{~cm}^{2}$, and piezoelectric fans with stainless steel blades were used. By cooling fins with $2 \mathrm{~W}$ input, the results showed that the piezo fans were effective up to $1500 \mathrm{~s}$ when placed at four different positions. The experimental error was around $8 \%$.

Li et al. ${ }^{(7)}$ studied heat transfer effects, and cooling and pressure decrease between transverse flows of plate-type radiators in a rectangular channel. In their study, it was discovered that an increase in the Reynolds number reduced the thermal resistance of a heat sink. Additionally, when the Reynolds number, height, and width of the radiator fins were increased, the pressure decrease also increased proportionally. However, when the Reynolds number reached a certain value, the heat transfer effects leveled off and did not improve further.

Liu et al. ${ }^{(8)}$ investigated the effect of horizontally and vertically stacked piezoelectric fans on the heat dissipation effects of heating blocks. During experimentation, six piezoelectric fans of different sizes and a heating block source measuring $45 \mathrm{~mm}$ along its long length were used. Results indicated that the enhancement of heat transfer was due to the jet stream from the tip of the blades and the entrained stream coming from both sides of the blades. The best heat transfer position for the horizontally stacked fan blades was a symmetrical distribution in the center; the best positions for the vertically stacked fan blades were unsymmetrical, located on the blade tip a quarter of the way above the heat source plane. The heat transfer performance attenuated significantly horizontally because of the blocking effect of the entrained air stream. However, not all the horizontally stacked fans showed poor heat transfer performance, which could be correlated with the distance to the plate surface. After analyzing the experimental results of the tests, an average deviation of $4.8 \%$ was used to describe the effect of geometric parameters in the case of the vertically stacked fans.

Petroski et al. ${ }^{(9)}$ tried different forms of cooling fins to compare the volume coefficient of performance with those of piezoelectric fans under $120 \mathrm{~V}$ and $60 \mathrm{~Hz}$, and then discussed the resultant radiator internal pressure loss and eddy current area. Heat transfer performance can be described using the volumetric coefficient of performance $\left(\mathrm{COP}_{\mathrm{v}}\right)$. The greater the amplitude of the fans, the smaller the heat resistance of the cooling fins. Moreover, the airflow velocity of piezo fan blades can reach $1.5 \mathrm{~m} / \mathrm{s}$, showing better heat dissipation performance than both ordinary natural convection and traditional axial flow fans.

Choi et al. ${ }^{(10)}$ used piezo fans with a frequency of $180 \mathrm{~Hz}$ and explored the vorticity from their oscillations by experimental and 2D numerical simulation approaches. For the numerical simulation study, the velocity intensity of 40 periods after the opening of the piezoelectric fans as grids was adopted for independent analysis. The flow field area determined from their numerical simulations was consistent with the experimental results. A numerical simulation method is sufficient to analyze the features of vorticity as a whole, and the trigger of vorticity caused by piezoelectric fans can be divided into the following four stages: initiation, development, separation, and propagation.

Abdulah et al., ${ }^{(11)}$ using both numerical simulation and experimental methods, concluded that the use of piezoelectric fans for electronic cooling had significant effects on heat transfer and 
flow fields. After changing the height of a piezoelectric fan and setting a heat source, their results reflected that a greater height showed a better heat transfer performance and reduced the heat source temperature.

Buermann et al. ${ }^{(12)}$ observed the flow field of a piezoelectric fan by a topology method, calculated the symmetrical disturbance caused by the front blades, and conducted a comparative analysis using a Bernoulli model and finite element methods. Their research revealed that the ratios of the cover length of piezoelectric ceramic materials in both blade length and blade thickness significantly affected the performance of the piezoelectric fan.

Acikalin et al. ${ }^{(13)}$ investigated convection heat transfer at various distances between the heat source and the blades. With a model for the heat flow field obtained using numerical simulation, the heat transfer efficiency of the piezoelectric fan reached as high as $375 \%$ compared with natural convection. The factors affecting the cooling efficiency were frequency, amplitude, and the distance from the heat source to the piezoelectric fan.

Acikalin and Garimella ${ }^{(14)}$ discussed the effects of piezoelectric fans from three different aspects. They conducted tests by changing the amplitude of the fan, the distance from the heat source to the fan, and the offset of the resonant frequency and length of the fan. A 2D model was established using Fluent software and the results were then compared with the experimental results of our study. When the frequency equaled the resonant frequency, the heat flow field was mostly affected. Furthermore, shorter blades were more effective than longer blades in heat dissipation performance when the amplitude remained the same. As for the distance from the heat source to the piezoelectric fan, the simulated results were similar to our experimental data when the distance was decreased, which meant that the actual flow field was equivalent to the simulated 2D flow field.

Sufian et al. ${ }^{(15)}$ investigated the heat produced by high-power light-emitting diode arrays and used a piezoelectric fan for cooling. The experiment demonstrated that a single piezoelectric fan helped increase heat transfer by about 1.8 times while two fans helped increase heat transfer by 2.3-2.4 times. In this work, a vertical piezoelectric fan was placed above the pin-fin heat sink and the boundary layer of the cooling fins broke through the impingement flow from the swing of the piezoelectric fan. The mixture of the surrounding cold air and hot air in the cooling fins moved heat away and thus changed the flow field structure of the pin-fin heat sink, which increased the heat transfer performance. In conclusion, piezoelectric fans are effective heat dissipation tools.

\section{Geometry}

\subsection{Calculation region and parameter setting}

The scope of the numerical simulation was set within a $300 \times 50 \times 100 \mathrm{~mm}^{3}(X \times Y \times Z)$ rectangular flow channel with piezoelectric fans installed inside for the actuation of square-columntype radiator fins, as shown in Fig. 1. The model framework was established on the basis of the number of grids to save time in numerical simulation calculations.

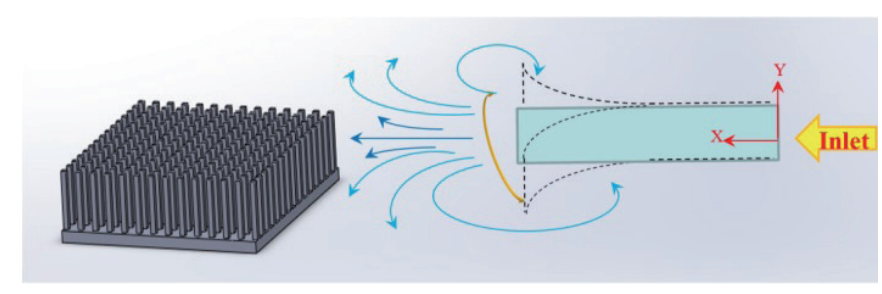

Fig. 1. (Color online) Schematic diagram of vertically stacked piezoelectric fans. 


\subsection{Governing equations}

The following governing equations were used in this study.

(1) Continuous equation:

$$
\frac{\partial \rho}{\partial t}+\nabla \cdot(\rho \vec{v})=0
$$

(2) Momentum equation:

$$
\frac{\partial}{\partial t}(\rho \vec{v})+\nabla \cdot(\rho \vec{v} \vec{v})=-\nabla p+\nabla \cdot(\overline{\bar{\tau}})+\rho \vec{g}+\vec{F}
$$

(3) Energy equation:

$$
\frac{\partial}{\partial t}(\rho h)+\nabla \cdot(\rho h \vec{v})=\nabla \cdot\left[\left(k+k_{t}\right) \nabla T\right]+S_{h}
$$

(4) Solid heat equation:

$$
\frac{\partial}{\partial t}(\rho h)=\frac{\partial}{\partial x_{i}}\left(k \frac{\partial T}{\partial x_{i}}\right)+S_{h}
$$

\subsection{Boundary conditions}

Before the analysis, the following assumptions were made for further discussion and research.

(1) The fluid is air.

(2) Transient state.

(3) Effects of gravity neglected.

(4) No changes with temperature and time.

(5) Laminar flow.

\section{Numerical Methods}

Standard and SIMPLEC rules were adopted for the pressure and pressure-velocity terms of the settings, respectively. Additionally, a first-order upwind scheme was used for the momentum equation and energy equation. Both velocity and pressure in the $X, Y$, and $Z$ directions were initialized and the convergence-condition values of velocity, momentum equation, and energy equation in the $X, Y$, and $Z$ directions were set. Then, calculations were conducted after the average temperature of the monitoring outlet was set. The convergence conditions were set as the scaled residual of the energy equation below $10^{-8}$ and the residuals of other equations were below $10^{-5}$.

When applying the first law of thermodynamics, we must first define that there is a certain area surrounded by the control surface in the given space and assume that energy and materials can be transferred in and out of this surface; this area is called a control volume. By using the heat conduction equation to represent the conservation of energy, its general formula can be written as

$$
\dot{E}_{\text {in }}+\dot{E}_{g}-\dot{E}_{\text {out }}=\dot{E}_{\text {st }}
$$


$\dot{E}_{g}$ stands for the heat produced in the process of energy conversion within the control volume. Since there is no energy conversion in this work, this term was omitted. $\dot{E}_{\text {in }}$ and $\dot{E}_{\text {out }}$ stand for the thermal energy and mechanical energy in and out of the control volume, respectively. $\dot{E}_{s t}$ stands for the change in physical internal energy as the internal energy of the control volume may also change. The rate of change of energy per unit of volume over time in Eq. (5) can be rewritten as

$$
\iiint_{C . V} \rho c_{p} \frac{\partial T}{\partial t} d x d y d z+h A_{t}\left(T_{\text {ave }}-T_{\infty}\right)-\dot{E}_{\text {in }}=0
$$

where $\rho$ and $C_{p}$ are density and specific heat, respectively, and their product is called volumetric heat capacity. $\frac{\partial T}{\partial t}$ is the rate of change of energy per unit of volume over time, $d x d y d z$ is the control volume, $A_{t}$ is the total heat radiator area, $h$ is the average convection heat transfer coefficient, and $\left(T_{\text {ave }}\right.$ $\left.-T_{\infty}\right)$ is the temperature difference between the control surface and the fluid volume.

The thermal resistance of heat convection can be applied to the heat convection of a surface, as defined by Newon's law of cooling. The most common method is to calculate the thermal resistance for the evaluation of heat transfer efficiency, which can be defined as

$$
R_{t h}=1 / h A_{t}
$$

The main goal of this work was to investigate the effect of the speed of wind generated by piezo fans on the heat transfer effects of the radiator. To accomplish this, grid encryptions for the surrounding area of the radiator and the swing area of the piezo fans were conducted and speed data in the $X$-axis were analyzed: every 20 cycles after launching the fans. Data capture points were set to be $22.5 \mathrm{~mm}$ ahead of the piezo fans. The total number of grids over the $X$-axis velocity with curve fitting is shown in Fig. 2, in which the grid numbers are $8 \times 10^{4}, 1.4 \times 10^{5}, 1.7 \times 10^{5}, 2$ $\times 10^{5}, 3.1 \times 10^{5}$, and $3.8 \times 10^{5}$. Fewer points can cause greater errors, while more points can lead to longer computing times; therefore, the number of grids in this work was $2 \times 10^{5}$ after taking both the accuracy of the model and the time cost into consideration.

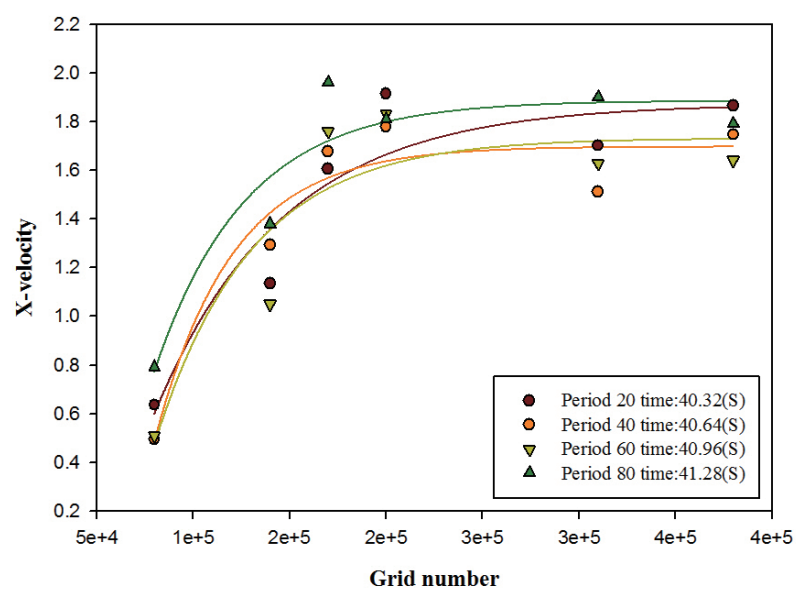

Fig. 2. (Color online) Fitted curves of total number of grids vs the $X$-axis velocity. 
In a numerical simulation, numerical stability and precision are dependent on the grid size and time step, as shown in Eq. (8). After setting the grid size, the control of the time step becomes important in the simulation. When the time step becomes relatively small, simulation times can increase considerably.

$$
C_{L}=\frac{u \times \Delta y}{\Delta L}
$$

$C_{L}$ is the current number, $\Delta L$ stands for grid size, $\Delta y$ stands for the calculated time step, and $u$ stands for fluid velocity.

If the time step in Eq. (8) is large, the calculation time for numerical simulation is short. However, this operation is less accurate and can lead to distortion or even negative volume for the grids. From the transient solution of a moving boundary, in this paper, we indicated that the flow field changed over time and the choice of time step affected the accuracy of the simulation. The time steps used in this work were $0.0002,0.0001$, and $0.00005 \mathrm{~s}$. The velocity changes over time in the $X$-axis were similar to those at 0.0002 and $0.00005 \mathrm{~s}$. In order to make the calculation for numerical simulation more efficient, $0.002 \mathrm{~s}$ was adopted as the time step in this work. The swing period $(0.016 \mathrm{~s})$ of the piezoelectric fans was divided into 80 time steps.

\section{Results and Discussion}

After heating for $40 \mathrm{~s}$, the piezoelectric fans were activated for $2 \mathrm{~s}$. The distance from the front of the fans to the front of the cooling fins $\left(L_{g}\right)$, the height distance of the piezo fans $\left(H_{w}\right)$, the width of cooling fins $\left(W_{f}\right)$, and the number of fins $(n)$ were calculated and discussed.

As shown in Fig. 3, data capture lines were arranged along the flow channel around the radiator in the front, rear, right, and left. In each direction, top-line, midline, and bottom-line data were taken and then used to show the changes in $H_{w}$ and $L_{g}$ vs velocity in the $X$-and $Z$-axes.

\subsection{Characteristics of piezoelectric fans in 2D flow field}

The flow field dynamic behavior of the piezoelectric fans were calculated in a 2D flow field using the computational fluid dynamics software FLUENT to obtain the boundary velocity field in the transient process and a 2D square surface. Changes in flow field were investigated after squarecolumn-type radiator fins were added inside the rectangular flow channel, by a 3D viewing method. Particles with sufficient density were placed in the velocity field and the changes in particle motion

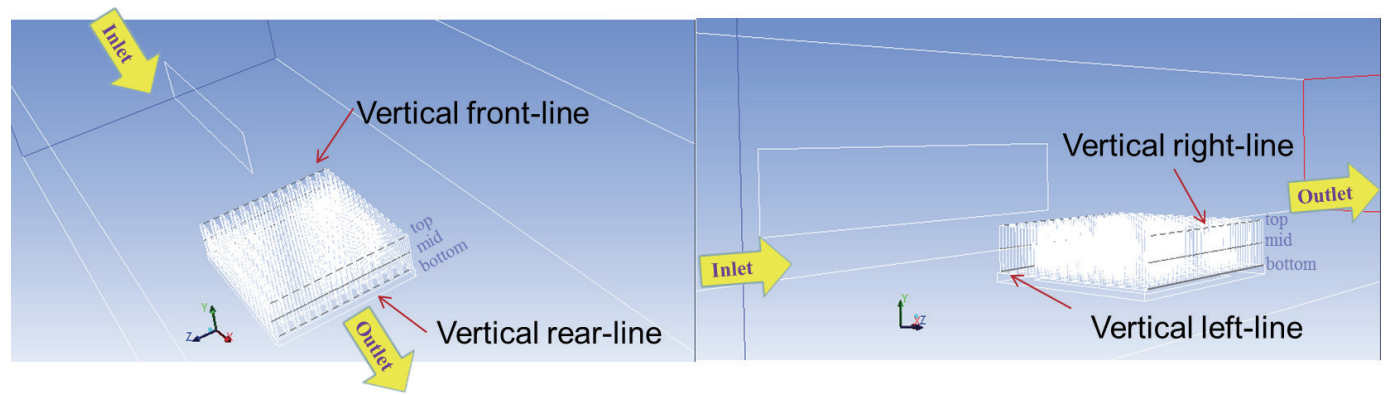

Fig. 3. (Color online) Schematic diagram of the arrangement of data capture lines around the flow channel of the radiator. 
at different times were observed. After the trends of the piezo fan flow fields were recognized, the 3D flow field was observed for further testing.

As shown in Figs. 4 and 5, during the period from 0.032 to $0.48 \mathrm{~s}, L_{g}$ was changed and the trends of the flow field were examined after the addition of square-column-type fins with the piezoelectric fans placed vertically. When $L_{g}=15 \mathrm{~mm}$, the fans actuated the formation of the flow field vortex. Owing to the long distance and the blocking effect of the square-column-type fins, the vortexes could only form constantly between the front of the fins and the piezo fans, and the velocity flow field that actually affected the field was very small. When $L_{g}=5 \mathrm{~mm}$, the impingement flow and the vortex from the blade tip were in a stable repeated overlay mode, effectively going inside the interior of the fins and considerably affecting them. As a result, the inner scope of the squarecolumn-type fins that the flow field entered increased with the decrease in $L_{g}$ value.

\subsection{Distance from the center of piezoelectric fan to the bottom of flow channel $\left(H_{w}\right)$}

When $L_{g}=5 \mathrm{~mm}, H_{w}$ changed, as shown in Fig. 6 . The other parameters were set as $W_{f}=1 \mathrm{~mm}$, $H_{f}=10 \mathrm{~mm}$, and the columns of radiator fins $n=14$, and the piezo electric fans were vertically placed. In Figs. 6(a)-6(d), when $H_{w}$ was in the range of 10 to $21 \mathrm{~mm}$, the image of the crosssectional temperature distribution at the fin height of $5 \mathrm{~mm}$ in the $Y$-axis was taken after the fans ran for $42 \mathrm{~s}$. Figure 6(a) shows that the heat dissipation performance was good when $H_{w}=10 \mathrm{~mm}$.

Figure 7 shows the distribution of velocity variation when $H_{w}=10 \mathrm{~mm}$ along the $X$-and $Z$-axes after the fans ran for $2 \mathrm{~s}$. On account of the configuration of the vertical piezoelectric fans, the heatradiating area became larger when the fans were closer to the bottom. The results in Fig. 7(a) reveal that the effect of the impingement flow on the front velocity was strong with the energy transfer of the top and midlines at the front of the radiator, and the fastest velocity change became around $2 \mathrm{~m} / \mathrm{s}$. The monitoring lines at the rear of the radiator were affected by the pressure difference and friction force when passing through the square-column-type radiator fins. Thus, the speed became lower as the fluid resistance in the rear became greater. The monitoring line at the left and right sides of the radiator became faster closer to the front of the piezoelectric fans, as shown in Fig. 7(b). During the blade swing process, the resistance above the fins was less and the monitoring line was at the top, showing higher speed than the midline and lower speed than the monitoring line at the front. Owing to the velocity gradient when the fluid was passing through a solid, the fluid velocity was zero at the interface; therefore, the speed at the bottom monitoring line was not discussed.

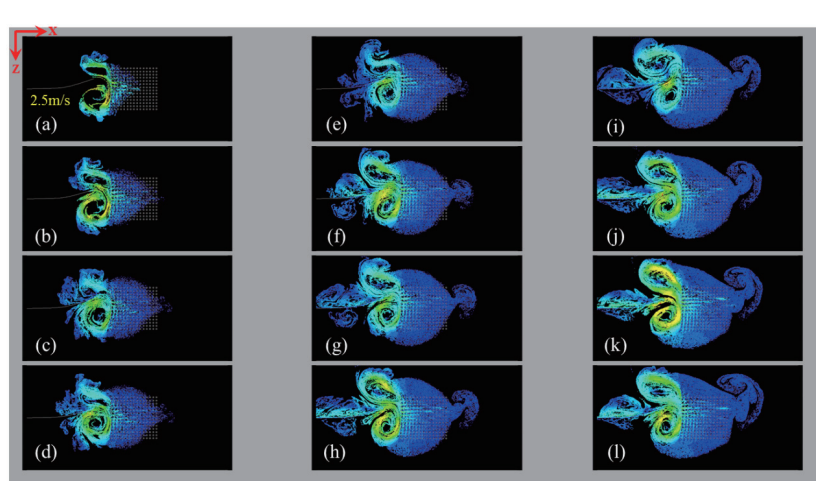

Fig. 4. (Color online) 2D piezoelectric fan configuration for $L_{g}=15 \mathrm{~mm}$.

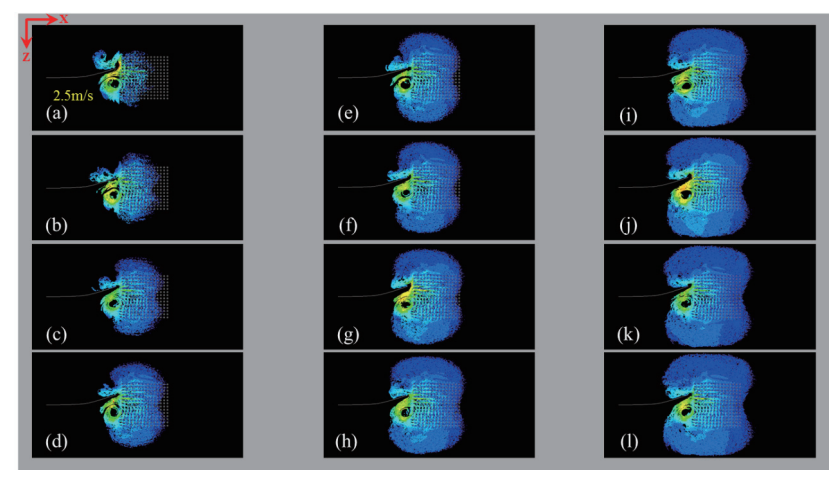

Fig. 5. (Color online) 2D piezoelectric fan configuration for $L_{g}=5 \mathrm{~mm}$. 

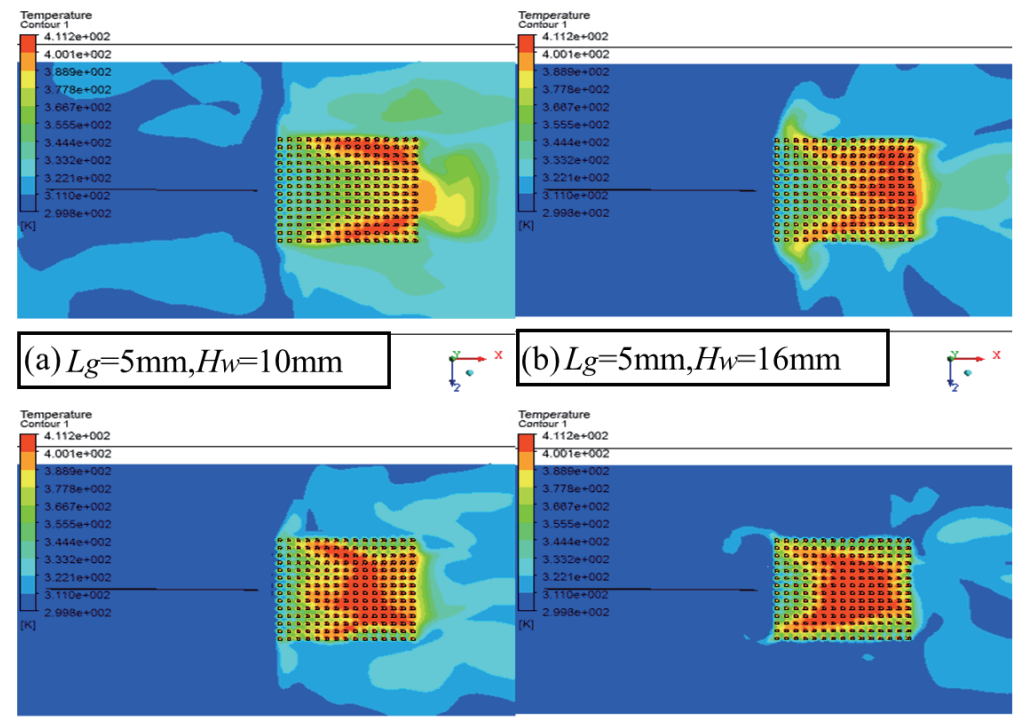

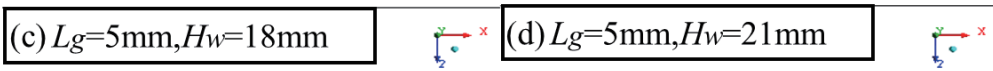

Fig. 6. (Color online) Cross-sectional temperature distribution with change in $H_{w}$.

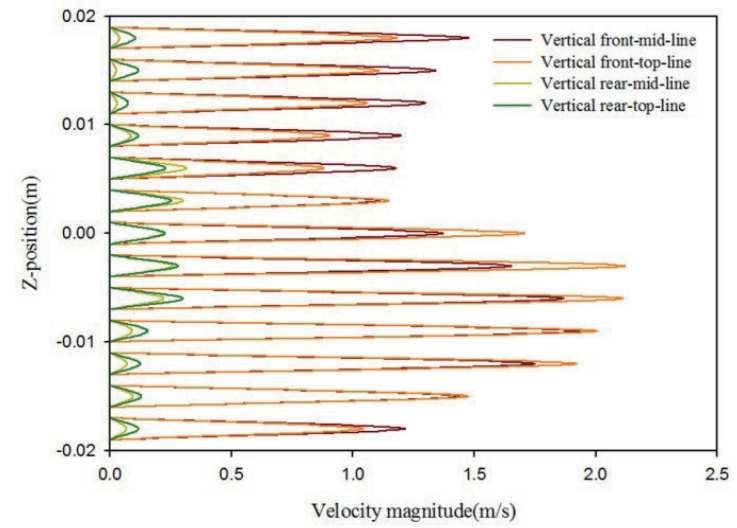

(a)

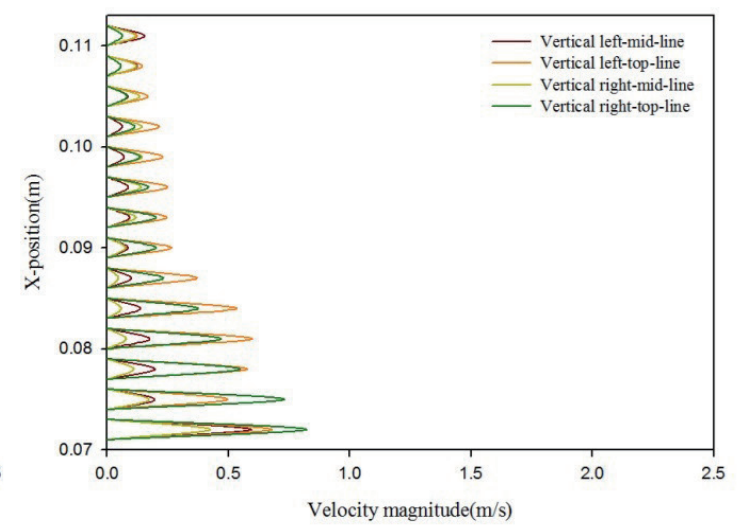

(b)

Fig. 7. (Color online) Distribution of velocity changes along (a) $X$ - and (b) $Z$-axes when $H_{w}=10 \mathrm{~mm}$.

Figure 8 shows the effect of changing $H_{w}$ on the temperature gradient. When the position of fans was farther away from the bottom plate, their effect on the radiator became weaker and the temperature gradient rose. When the position of the fans was $H_{w}=10 \mathrm{~mm}$, which was the lowest position in the model, a rather low temperature gradient was achieved. However, when the position was $H_{w}=21 \mathrm{~mm}$, the temperature gradient clearly increased. The impact of the piezoelectric fans could not affect the inside the fins of the radiator, so the entrained flow did not have a large impact on the thermal boundary layer of the radiator fins, which in turn lead to the rapid increase in the temperature gradient. After the launch of the piezoelectric fans, the temperature gradient trend gradually became clear at the operation frequency of the fans $(62.5 \mathrm{~Hz})$. 


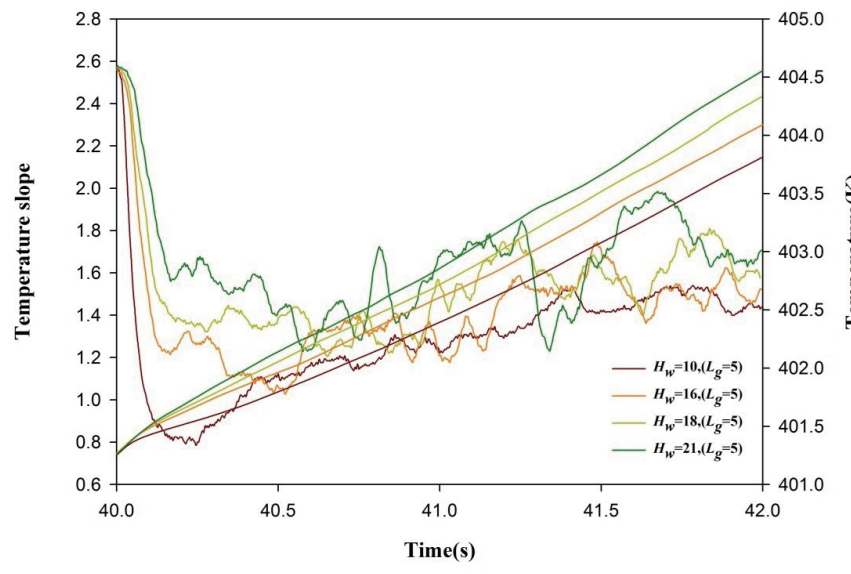

Fig. 8. (Color online) Effect of changing $H_{w}$ on the temperature gradient.

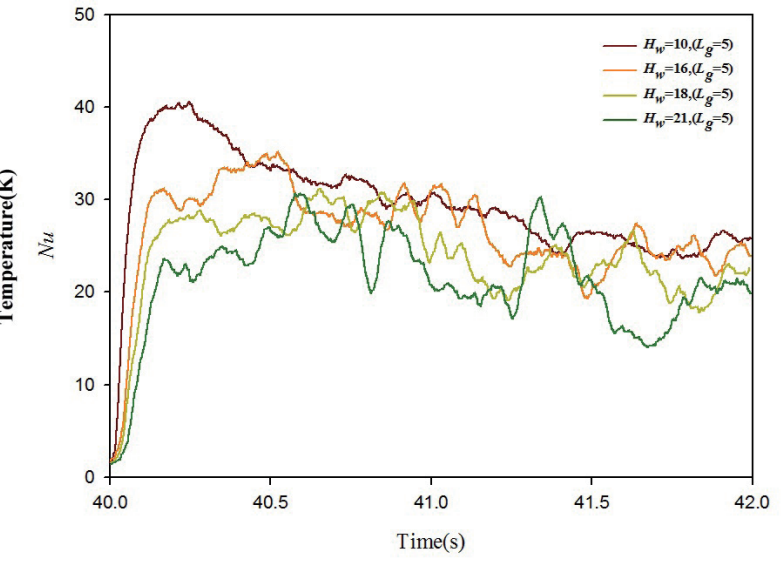

Fig. 9. (Color online) Effect of changing $H_{w}$ on the Nusselt number $N_{u}$.

Figure 9 shows the effect of changing $H_{w}$ on the Nusselt number $N_{u}$. The average heat convection coefficient increased with increasing temperature difference between the radiator surface and the environment. The larger the temperature difference, the steeper the temperature gradient. The impingement flow forming at the front side of the blades when $H_{w}=10 \mathrm{~mm}$ had a greater impact on the radiator. As the impingement flow effectively formed a velocity boundary layer within the radiator, the convective heat transfer value on the radiator fins was relatively high.

\subsection{Distance from the front side of piezoelectric fan blades to the front side of radiator $\left(L_{g}\right)$}

Figure 10 illustrates the effect the changes in $L_{g}$ had on the thermal resistance. A lower resistance meant that the position of the fans had a greater effect on the heat dissipation effects of the square-column-type radiator fins. The lowest resistance was observed when $L_{g}=-22.5$ $\mathrm{mm}$. The fans were placed vertically and the impingement flow was above the heat source of the baseplate. This advantage became more obvious when the square-column-type radiator fins were used for heat dissipation. In contract, when $L_{g}=5 \mathrm{~mm}$ and $L_{g}=-45 \mathrm{~mm}$, the farther from the center of the blade tip to the center of the heating plate, the greater the thermal resistance.

Figures 11(a) and 11(b) show temperature charts with the fans vertically placed for $L_{g}=5$ and $-45 \mathrm{~mm}$. In Fig. 11(a), the piezoelectric fan was placed at $L_{g}=5 \mathrm{~mm}$, which was a short distance from the front of the fins. Radial lines presented on the fins were mostly in the form of impingement flow. By taking the cross-sectional temperature distribution with the fin height $H_{f}$ of $5 \mathrm{~mm}$ for analysis, it was discovered that the effect of the piezoelectric fans with $L_{g}=5 \mathrm{~mm}$ was clearer. However, when $L_{g}=-45 \mathrm{~mm}$, the vortex easily formed in the front, left, and right sides because of the lack of fin blocking. As a result, the temperature boundary layer at the back of the fins could be effectively broken by the formation of a vortex. As shown in Fig. 12(b), the cross-sectional temperature distribution at the fin height $H_{f}=5 \mathrm{~mm}$ along the $Y$-axis was used for analysis. This revealed that the effect of the piezoelectric fans with $L_{g}=-45 \mathrm{~mm}$ was clearer but the inner heat of the square-column-type radiator fins could not be effectively dissipated with either $L_{g}=5$ or $-45 \mathrm{~mm}$. This explained why the resistance was higher at these two positions. 


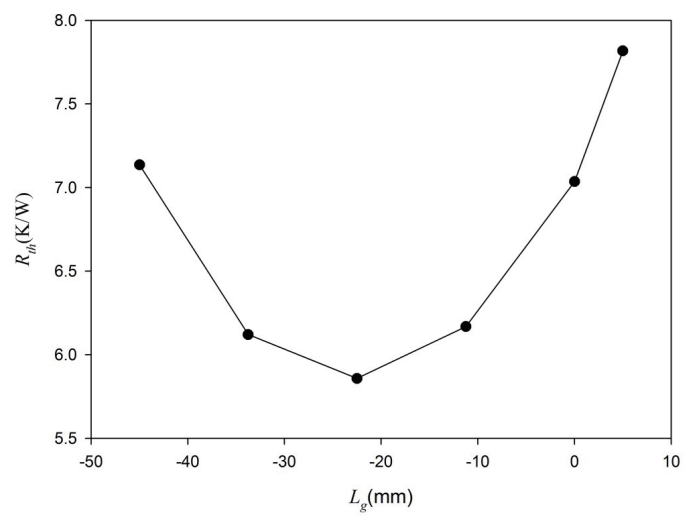

Fig. 10. Effect of changing $L_{g}$ on thermal resistance.

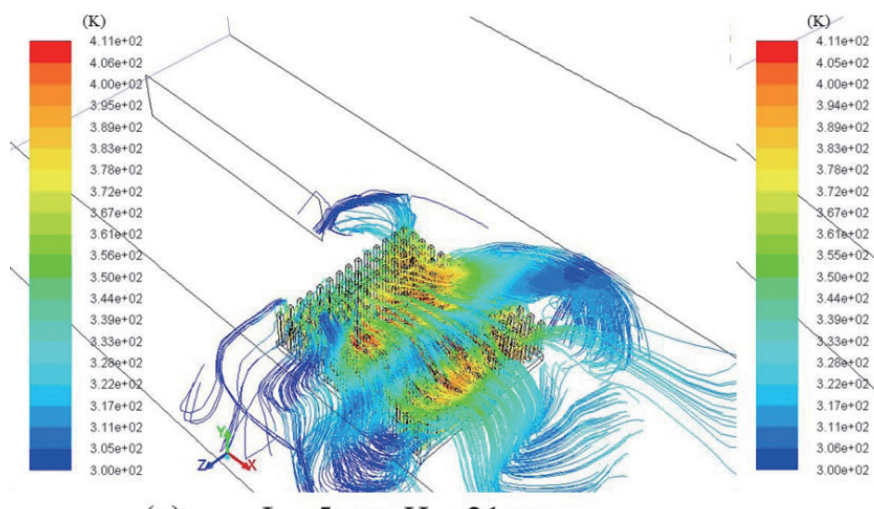

(a) $\quad L g=5 \mathrm{~mm}, H w=21 \mathrm{~mm}$

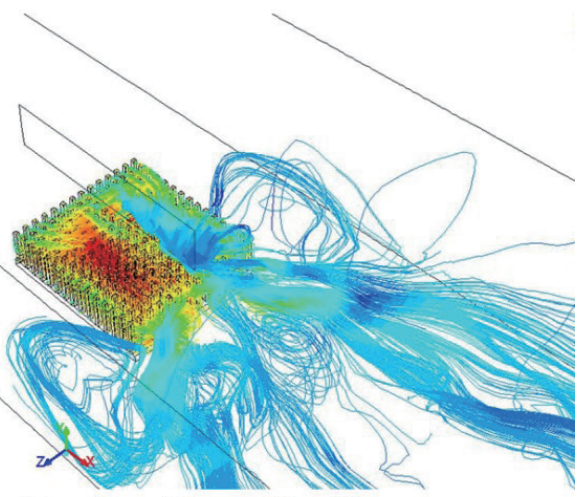

(b) $L g=-45 \mathrm{~mm}, H w=21 \mathrm{~mm}$

Fig. 11. (Color online) Temperature radial lines when $L_{g}=$ (a) 5 and (b) $-45 \mathrm{~mm}$.

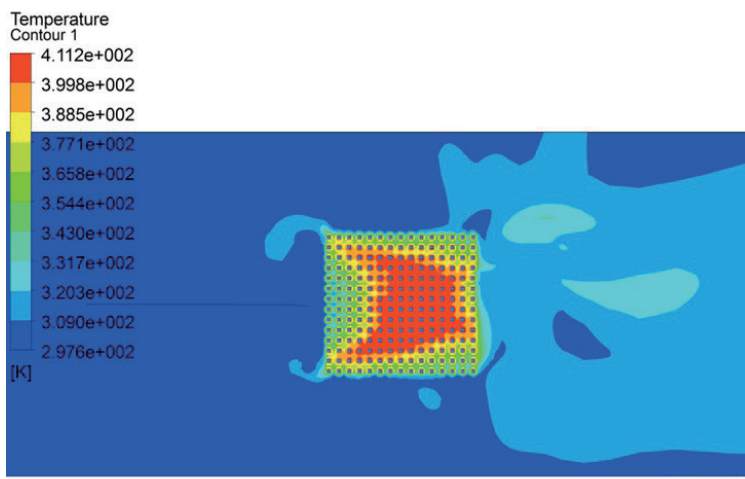

(a) $L g=5 \mathrm{~mm}, H w=21 \mathrm{~mm}$

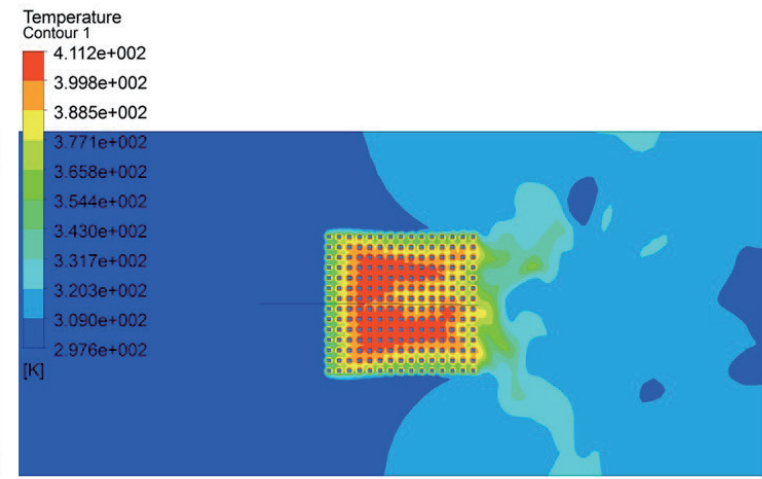

(b) $L g=-45 \mathrm{~mm}, H w=21 \mathrm{~mm} \quad \longrightarrow$

Fig. 12. (Color online) Cross-sectional temperature distribution along $Y$-axis.

\subsection{Effect of changing the number of cooling fins}

Figure 13 shows the effect of changing $H_{w}$ on the thermal resistance in fin columns $n=10$ and $n$ $=14$. The piezoelectric fans were vertically placed and the distance from the front of the blades to the front of the radiator was fixed $\left(L_{g}=5 \mathrm{~mm}\right)$. Since column $n=14$ had a larger cooling area and a 
smaller fin spacing $(G)$ than column $n=10$, the wind could not reach the inside of the cooling fins. Therefore, the thermal resistance of the column $n=10$ was lower than that of column $n=14$.

From the perspective of the $Y$ - and $Z$-axes, the arrangement of the vertical piezoelectric fan and the lowest thermal resistance of the fin column were observed. The velocity vector distribution was depicted with the heat flux located from the center of the piezoelectric fan in column $n=14$ to the bottom of the flow channel with $H_{w}=10 \mathrm{~mm}$ and from the center of the piezoelectric fan in column $n=10$ to the bottom of the flow channel with $H_{w}=16 \mathrm{~mm}$. As shown in Fig. 14, the heat flux performance was most obvious on the front side of the fins when $n=14$ and $H_{w}=10 \mathrm{~mm}$, and the piezoelectric fan was placed vertically. Because of the relatively small fin spacing, the fluid velocity in the blade tips had an impact on the front of the cooling fins, causing the cooling fins to exert a counterforce to shock the fluid. The resultant impingement flow moved along the direction of the $Z$-axis owing to the blocking effect of the radiator fins in the front. In order to show this phenomenon more clearly, a piezoelectric fan was placed vertically in front of the fins. The flow field formed a fan-shaped extension from the perspective of the $X$ - and $Z$-axes, as shown in Fig. 15. However, owing to the accumulation of heat, heat transfer was very limited, and the outer side of the fan-shaped section demonstrated a lower heat transfer rate.

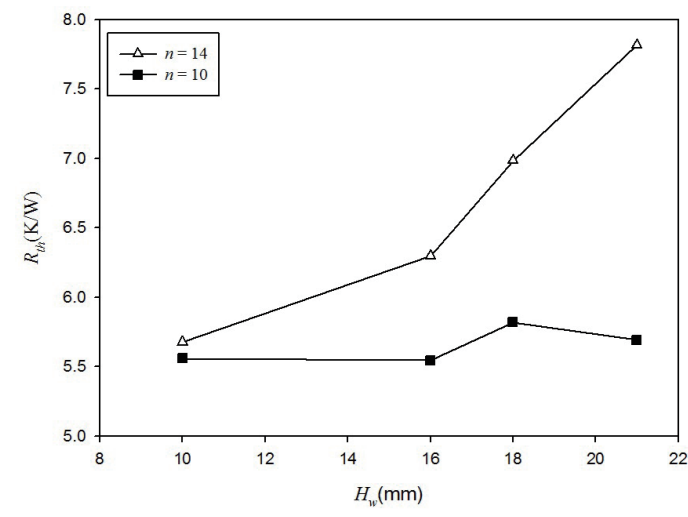

Fig. 13. Effect of changing $H_{w}$ on thermal resistance.

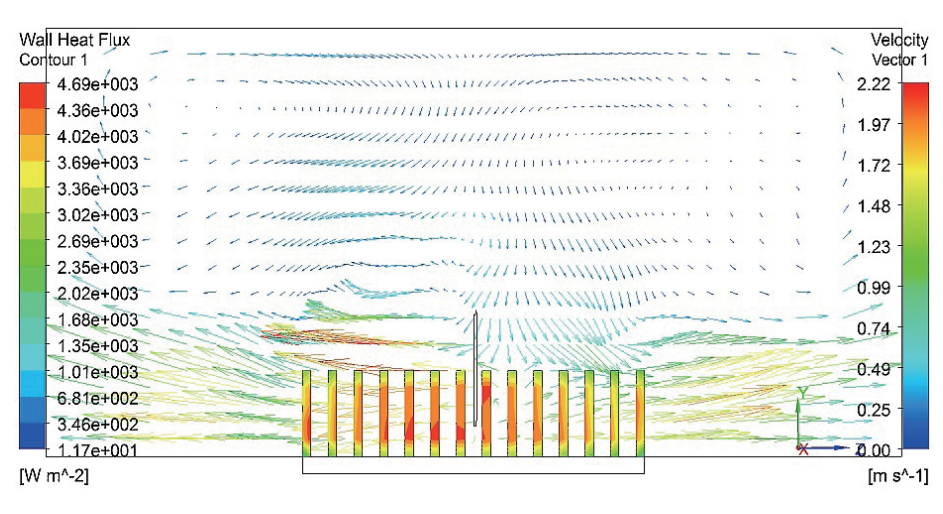

Fig. 14. (Color online) Heat transfer rate and velocity vectors along $Y$-axis.

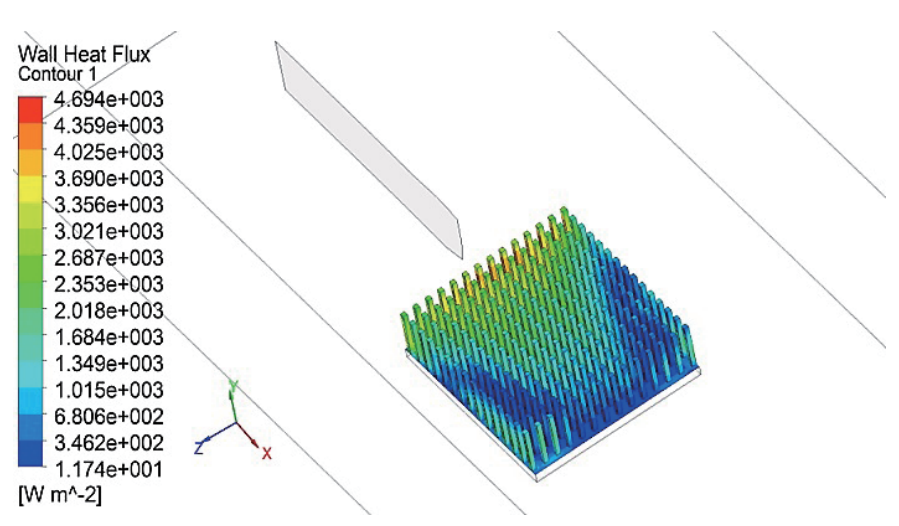

Fig. 15. (Color online) Fin column with $n=14, H_{w}=10$, and $L_{g}=5$. 


\section{Conclusion}

With a piezoelectric fan placed vertically in front of the square-column-type radiator fins, the velocity flow field generated during the swing process of blades was able to break the radiator speed boundary layer. This allowed the flow field to move into the interior of the radiator efficiently. As for the radiator itself, a blast of impingement flow towards the front and bottom sides could be formed to remove the heat accumulated inside the radiator by using the piezoelectric fan to flap and disrupt the flow field. This phenomenon was most obvious in column $n=10$ of the cooling fins, and was related to the fin spacing $(G)$. The effect of the blocking effects of the radiator flow channel on the velocity of the impingement flow resulted in a staggered change in flow field velocity inside the radiator, which was most obvious as the heat accumulation phenomenon of fins. Moreover, by making use of columns to block fluid and with the effects of the piezoelectric fan, both the mixture of fluid and heat transfer performance were considerably improved.

\section{References}

1 M. Toda: Ferroelectrics 22 (1979) 911.

2 T. Acikalin, S. M. Wait, S. V. Garimella, and A. Raman: Heat Transfer Eng. 25 (2004) 4.

3 P. Bürmann, A. Raman, and S. V. Garimella: IEEE Trans. Components and Packaging Technologies 25 (2003) 592.

4 S. Naik, S. D. Probert, and I. G. Bryden: Int. J. Heat Fluid Flow 20 (1999) 374.

5 S. A. El-Sayed, S. M. Mohamed, A. M. Abdel-latif, and A. E. Abouda: Exp. Therm. Fluid Sci. 26 (2002) 879.

6 M. Choi, C.Cierpka, and Y.-H. Kim: J. Fluids Struct. 31 (2012) 67.

7 M. K. Abdullah, M. Z. Abdullah, S. F. Wong, C. Y. Khor, Y. Ooi, K. A. Ahmad, Z. M. Ripin, and M. A. Mujeebu: 10th Int. Conf. Electronics Material and Packaging (2008) p. 165.

8 H.-Y. Ju , I.-H. Jae, and C. Wenwn: Sens. Actuators 79 (2000) 8.

9 T. Acikalin, A. Raman, and S. V. Garimella: Acoust. Soc. Am. 114 (2003) 1785.

10 M. Kimber, S. V. Garimella, and A. Raman: J. Heat Transfer 129 (2007) 1168.

11 K. H. Tseng, M. Mochizuki, K. Mashike, T. Nguyen, F. Kiyoka, and T. Kosakabe: 2nd Int. Forum Heat Transfer, Tokyo, Japan, Sep. 17-19, 2008.

12 J. Petroski, M. Arik, and M. Gursoy: IEEE Trans. Components and Packaging Technologies 33 (2010) 25.

13 M. Choi, S. Y. Lee, and Y. H. Kim: Eur. J. Mech. B 34 (2012)146.

14 M. Choi, C. Cierpka, and Y. H. Kim: Eur. J. Mech. B 43 (2014)154.

15 S. F. Sufian, M. Z. Abdullah, and J. J. Mohamed: Int. Commun. Heat Mass Transfer 43 (2013) 81.

16 S. F. Sufian, Z. M. Fairuz, M. Zubair, M. Z. Abdullah, and J. J. Mohamed: Microelectron. Reliab. 54 (2014) 1534. 